\title{
Modelo de evaluación del desempeño laboral para el personal administrativo universitario.
}

Model of evaluation of work performance for university administrative staff

\author{
Alvarez Indacochea Blanca Viviana ${ }^{(1)}$ \\ Indacochea Ganchozo Blanca Soledad ${ }^{(2)}$
}

Yoza Rodríguez NarcisoRoberto ${ }^{(3)}$

Alvarez Indacochea Arturo Antonio ${ }^{(4)}$

(1), (3) y (4) Facultad de Ciencia Económicas del área Administrativa de la Universidad Estatal del Sur de Manabí.

(2)Vice-Rectora. Universidad Estatal del Sur de Manabí. Ecuador

Contacto: vivialvarez02@hotmail.com

Receptado: 19/02/2018

Aceptado: 16/04/2018

\section{Resumen}

La evaluación del desempeño laboral para el personal administrativo de la educación superior es un proceso complejo, porque se requiere ejercer actividad laboral que correspondan a los niveles precisos de conocimientos en el puesto de trabajo asignado para esto se necesita como pasos previos la definición de perfiles ocupacionales, estructurados en torno a conocimientos, habilidades, conductas individuales y sociales respuesta al desafío que implica, desde la perspectiva laboral con aspectos propios del trabajador, el objetivo de esta investigación es analizar la importancia de un modelo de evaluación del desempeño laboral con énfasis para el personal administrativo de la educación superior que contribuye a mejorar el desarrollo profesional del trabajador en aras de cumplir los objetivos y metas propuesta por la institución.

Palabra clave: Modelo, Evaluación, Desempeño, Educación superior

\section{Summary}


The evaluation of work performance for administrative staff of higher education is a complex process, because it is required to exercise work that correspond to the precise levels of knowledge in the job assigned for this is needed as previous steps the definition of occupational profiles, structured around knowledge, skills, individual and social behaviors, responding to the challenge that implies, from a work perspective with aspects of the worker, the objective of this research is to analyze the importance of a model of evaluation of work performance with emphasis for the administrative staff of higher education that contributes to improving the professional development of the worker in order to meet the objectives and goals proposed by the institution.

Keyword: Model, Evaluation, Performance, Higher education

\section{Introducción}

En la actualidad las evaluaciones del desempeño laboral en las instituciones públicas deben responder a las necesidades del trabajador; es por eso que se vuelve necesario contar con un personal altamente calificado, motivado y eficiente, y que se realice un buen direccionamiento de la toma de decisiones basadas en herramientas administrativas, por lo tanto es importante evaluar y fortalecer el desempeño laboral del personal administrativo que permita mejorar los resultados de los objetivos y metas planteadas por la institución.

La evaluación del desempeño laboral es un proceso que consiste en medir el nivel de eficiencia y eficacia del desempeño del empleado en su puesto de trabajo, así como las características y habilidades que éste debe tener en el mismo. Con todo lo ante expuesto, en la presente investigación se analizó la importancia de un modelo de evaluación que contribuya a fortalecer el desempeño laboral del personal administrativo de las universidades.

\section{Desarrollo}

\section{Marco conceptual de modelo de gestión de evaluación del desempeño laboral.}

Una definición generalizada del modelo, originada en el ámbito de evaluación del desempeño laboral es una representación simplificada de la realidad en la que aparecen algunas propiedades de los sistemas de la evaluación del desempeño laboral que pretende reproducir objetos que constituyen conceptos o conjunto de relaciones que utilizan para representar y estudiar de forma simple y comprensible la realidad empírica. 


\section{Modelo}

Un modelo es la representación concisa de una situación; por eso representa un medio de comunicación más eficiente y efectivo. Se puede definir modelo como: La representación de una idea que sea utilizada como una guía de acción para organizar, explicar, entender o mejorar un sistema.

\section{Evaluación}

La evaluación del desempeño laboral es el proceso mediante el cual se miden las habilidades y destrezas de un trabajador en su puesto de trabajo, teniendo en cuenta actitudes y aptitudes relevantes con compromiso y aportación activa, capaz de generar una expectativa permanente de aprendizaje, innovación, desarrollo personal y profesional, en aras de cumplir con las metas y objetivos propuesto de forma individual, colectiva y organizacional.

Para (Delhumeau et al., 2013) la construcción de un modelo de gestión para la evaluación del desempeño laboral, es fundamental, sin el compromiso de sus integrantes o autor no sería posible el cumplimiento de sus metas y objetivos planteados basados en su eficiencia y eficacia de sus trabajadores. Con la participación de todos los implicados, se puede lograr un diálogo y consenso en la toma de decisiones, un clima laboral basado de confianza y esto nos llevaría a una motivación sostenida en las organizaciones.

Para Villegas, (1988) El modelo de Gestión de evaluación del desempeño laboral “consiste en un examen metódico del desempeño de la persona en el trabajo, para evidenciar capacidades, debilidades y realizaciones que inciden directamente sobre la productividad”. Es decir, que es un proceso necesario en la actividad administrativa, por medio del cual se pueden detectar las capacidades y debilidades en el desempeño laboral de los trabajadores, e implantarse planes adecuados para las necesidades que posean los mismos.

Según Arias, (2006) expone que se "reflejan los avances y el estado actual del conocimiento en un área determinada y sirven de modelo o ejemplos para las futuras investigaciones", es decir, se refieren a los estudios previos que guardan relación con el objeto de investigación.

García et al. (2008) proponen un modelo de evaluación por competencias a partir del conocimiento de prácticas de evaluación del desempeño, la experimentación de estrategias novedosas y diferentes indicadores de competencia para llegar a un resultado, como son: 
1. Cognitivos (adquirir y usar conocimiento para solucionar problemas).

2. Técnicos (habilidades, puesta en práctica de procedimientos).

3. Integral (integración de conocimiento básico y aplicado).

4. Relacional (comunicación efectiva).

5. Afectivo-moral (respeto ante una persona o situación).

Min-pemg et al. (2012) plantean un modelo para evaluar al personal de una de las áreas más importantes de la empresa. En el modelo se menciona que lo clave es establecer unos indicadores de rendimientos basados en la moralidad, habilidad, diligencia y utiliza AHP (Analytical Hierarchical Process) para determinar el peso de cada indicador.

Hablar de los modelos de evaluaciones laboral es de remontarnos hasta las épocas más antiguas de la humanidad, a través de un pasaje histórico se puede mencionar el uso de las características físicas de los sujetos tales como estatura, peso y complexión para la elección de los líderes de las tribus nómadas, estas evaluaciones, si bien superficiales servían como punto de referencia para tener una idea concreta de las capacidades a desempeñar. Conforme el paso del tiempo las evaluaciones de desempeño laboral se fueron haciendo más elaboradas, pues la exigencia y la demanda de una capacidad competente para ocupar un puesto o realizar una actividad determinada como requisito primordial.

\section{Importancia de los modelos de evaluación del desempeño laboral.}

La evaluación del desempeño laboral constituye un aspecto básico para la gestión óptima de las organizaciones y del ser humano, tomando en cuenta las necesidades del individuo orientadas a los objetivos de la organización, a través de acciones correctivas que alienten un desempeño satisfactorio.

Actualmente, las organizaciones en transformación saben que el ser humano asociado con otros, debidamente constituidos y con los recursos adecuados, es capaz de convertir en realidad las metas compartidas; es por eso que el modelo personal va siendo sustituido por la Gerencia del Talento Humanos, ante esta realidad, la mentalidad de los empresarios entienden que: administración de personal no es otra cosa que decidir acerca del conjunto de normas y reglas prácticas que tratan cómo las nuevas responsabilidades que iban descubriendo: como el clima laboral, en la actualidad conocido como clima organizacional, diseño de planes de sucesión, modernización de los sistemas retributivos, comunicación interna, motivación, calidad de vida, formación de los trabajadores, desarrollo de sus competencias. 
La administración de personal no es otra cosa que decidir acerca del conjunto de normas y reglas prácticas que tratan cómo dirigir y organizar al hombre en su trabajo y que las actitudes hacia las personas son más importantes para la eficiencia y la productividad que los propios factores materiales tales como: salarios al-tos, ambientes acogedores y horarios de trabajo menos recargados (Casimiro, 2007).

Hay características distintivas de cada modelo sin embargo todas conducen a la importancia de gestionar el proceso de ED como elemento integrador para una buena GTH en las organizaciones, estos elementos tributan a:

1. Objetivos de referencias, medidas comparativas y efectos resultantes.

2. Destaca la importancia de aplicar la metodología cualitativa para el estudio del objeto a evaluar en su entorno natural.

3. Favorece el desarrollo social, individual y colectivo.

4. Mejorar el desempeño de los empleados.

5. Mide sus resultados mediante las cuatro "c" (compromiso, competencia, congruencia, y costos eficaces).

6. Impulsa la responsabilidad social de la organización mediante el desarrollo de alianzas con las partes interesadas incrementando la creatividad y motivación de las personas de la organización

7. Facilita la alineación de la gestión del talento humano a través de las competencias.

8. La administración se vuelve herramienta central en la gestión del talento humano.

9. La identificación a los puntos débiles, permitiendo intervenciones de mejora que garantizan los resultados.

10. Brinda credibilidad a los departamentos de capacitación.

11. Brinda una metodología de elaboración real de las contribuciones y retorno financiero de los programas.

12. Desarrollo de las competencias de los trabajadores.

13. Toma en cuenta los factores causales para el éxito y fracaso.

14. Definición de indicadores de evaluación del desempeño.

15. Entrenamiento a evaluadores.

16. Retroalimentación y seguimiento.

La evaluación del desempeño permite la determinación y el desarrollo de políticas adecuadas a las necesidades de la organización. Es decir, la evaluación de los empleados consiste en evaluar 
la calidad de su desempeño, en la obtención de los resultados que son de su responsabilidad. Es por ello que se hace necesario que gerentes, directores, coordinadores y supervisores entiendan de la importancia de la necesidad de evaluar el rendimiento o desempeño de los empleados; unos y otros necesitan de su retroalimentación sobre sus esfuerzos.

El éxito o el fracaso de la Evaluación del Desempeño en la organización dependen de la filosofía utilizada para establecerla, las actitudes del personal a nivel Gerencial, de Dirección, Coordinación y Supervisión hacia el programa y su capacidad para cumplir con los objetivos y planes.

En tal sentido las políticas generales de las organizaciones en la evaluación del desempeño se deben basar en:

1. Que todas las organizaciones deben tener un único sistema de Evaluación del Desempeño, y este debe ser liderado por el departamento encargado de la administración del talento humano, quienes deben brindar asesoría, soporte y el apoyo necesario para su implementación y aplicación en todas las áreas de la Organización.

2. Ser una herramienta que permita analizar los logros, aportes, fortalezas y áreas de mejoramiento de los funcionarios de la organización en su desarrollo personal y profesional, con el fin de crear e implementar estrategias para el desarrollo y capacitación del talento humano y así preparar a la organización para competir dentro del sector de su actividad.

3. Efectuar la evaluación del desempeño sobre resultados directamente atribuible al trabajo individual y personal y al colectivo.

La dirección del talento humano, en dependencia del modelo utilizado, tendrá una herramienta que le brindaran unos resultados que no son el final del proceso, por el contrario, son el inicio de un plan de mediación transversal que abarcará toda la organización tanto de los equipos de trabajo como a las personas, permitiendo así que se ajusten los comportamientos y desempeños que se esperan en toda la organización según los resultados de los participantes en el proceso de evaluación.

Lo analizado hasta aquí permitirá evaluar las realidades y contextos del campo de acción a investigar en aras de proponer un modelo que responda a las políticas anteriores. 
Después de haber revisado los enfoques bibliográficos de diversos autores sobre los temas de importancia para el desarrollo de esta investigación, podemos observar que a través de la historia la gestión del talento humanos ha sufrido transformaciones en todos sus procesos sistemáticos, los mismos que han conducido a la formulación de diversos modelos de evaluación de desempeño laboral, y que deben considerarse como estratégico, en tanto pretende la mejor elección, educación, organización y satisfacción en la labor que realizan y el mayor rendimiento, estimulando el desarrollo de competencias específicas que tiendan a un desempeño laboral.

\section{Procesos de la evaluación del desempeño laboral.}

Dentro del proceso de la Evaluación de Desempeño HayGroup, (2006). considera que la evaluación de desempeño es un proceso de mejora continua de la gestión y eficiencia de la empresa y la base se encuentra en la comunicación integradora y en la evaluación permanente de cumplimiento de objetivos, en esta definición el autor establece que el proceso de evaluación de desempeño se rige mediante tres procesos los cuales que se tiene que determinar cuáles van hacer las funciones que va a desarrollar cada empleado las mismas que tienen que ir de acuerdo al perfil profesional, se debe hacer referencia de cuáles son las metas planteadas las mismas que tienen que ir acorde a los objetivos y misión de la Institución.

Por su parte para Seijas, (2011). Quien cita a Robbins, (2003). Proponen una serie de pasos que pueden ser de utilidad en la planificación de la evaluación; a continuación, se presenta la explicación de cada uno de ellos, según la autora:

- Preparar con anticipación el programa de la evaluación: es sumamente importante que tanto el evaluado como el evaluador se enteren con suficiente antelación que se pondrá en marcha este proceso para que tengan la oportunidad de prepararse, así se podrá sacar el máximo provecho del mismo.

- Crear un ambiente de apoyo que tranquilice a los empleados: los trabajadores deben sentirse relajados y actuar como lo hacen normalmente en sus labores, para que se pueda evaluar su desempeño real, esto no siempre es sencillo de lograr y es necesario que se haga todo el esfuerzo necesario para este fin. 
- Proceder a describir el propósito de la evaluación de los empleados: se debe ofrecer a los trabajadores toda la información acerca de para qué se utilizarán los resultados de su evaluación, para que tenga una visión clara del proceso y sus expectativas sean realistas.

- Realizar diálogo en las conductas de trabajo, no en los empleados: no es beneficioso que se ataque al trabajador, reprochándole las debilidades que ha presentado en su desenvolvimiento, más bien se debe exponer claramente las conductas en que ha incurrido que no contribuyen al logro de un mejor desempeño.

- Apoyar la evaluación con ejemplos específicos: para mayor claridad en la exposición de las fallas encontradas, es vital que se citen ejemplos de eventos en los cuales el trabajador se haya desempeñado por debajo de los niveles esperados.

- Proporcionar la respectiva retroalimentación positiva y negativa: en la evaluación se encontrarán, además de debilidades, aspectos positivos del desempeño del trabajador los cuales se deben afianzar a través del refuerzo positivo; informándole qué actividades ha llevado a cabo de manera adecuada obtendrá una visión global de su desenvolvimiento.

- Es vital que la evaluación del desempeño sea un evento que no se encuentre aislado de la planificación de la organización, sino más bien que sus bases sean las directrices en función de esto se planifique el proceso.

Otro proceso de evaluación del desempeño es el propuesto por Ivancevich (2005), el cual se compone de seis pasos que se enumeran a continuación:

\section{Establecer estándares de desempeño para todas las posiciones y los criterios de} evaluación: Basándose en los resultados arrojados por un análisis de puestos en la organización, que contenga las dimensiones del desempeño y los estándares que se esperan de los ocupantes; se constituyen criterios de evaluación que permitan valorar los niveles de rendimiento los ocupantes de los cargos, más no su personalidad, para alcanzar este fin es necesario que los criterios sean confiables, pertinentes, sensibles y factibles.

2. Establecer políticas de evaluación de desempeño sobre cuándo calificar, con qué frecuencia y quien debe hacerlo: La periodicidad de la evaluación del desempeño debe establecerse de acuerdo al ciclo de actividades de la empresa, a la naturaleza de la actividad a la que se dedique y al criterio propio de sus directivos, puede tomarse como referencia la fecha de inicio del trabajador en la organización y evaluarlo cada año en esa fecha o se puede fijar una fecha única para todos los trabajadores. 
3. Pedir a los evaluadores que reúnan datos sobre el desempeño de los empleados: los evaluadores destinarán un tiempo a la recolección de datos que les serán de gran utilidad en el momento de la aplicación del método de evaluación seleccionado, antes del cual es preciso que se capacite a los evaluadores, para que estén mejor preparados para recaudar la información necesaria.

4. Pedir a los evaluadores que evalúen el desempeño de los empleados: una vez que se ha elegido el método más idóneo y se ha informado tanto a los evaluadores como al trabajador acerca de los criterios e instrumentos que servirán para la evaluación del desempeño, se pide que se lleve a cabo dedición y estudio de la actuación de los trabajadores utilizando los instrumentos seleccionados.

5. Analizar la evaluación con el empleado: el diálogo es un componente mente importante del sistema de evaluación del desempeño, por lo cual debe haber una comunicación activa sobre el desempeño entre el supervisor y el subordinado; a través de una entrevista de revisión para analizar la evaluación y fijar los objetivos para el siguiente periodo, es muy importante que se de retroalimentación tanto negativa como positiva para que se pueda obtener una visión objetiva.

6. Tomar decisiones y archivar la evaluación: dependiendo de cuál sea la utilidad y el alcance que la organización le dé a la evaluación del desempeño, podrá utilizar los resultados obtenidos para tomar decisiones en cuando a: remuneraciones, capacitación, movimientos de personal (traslados, ascensos, transferencias), desincorporaciones, entre otros.

Como se puede observar existe una gran variedad de proceso de evaluación del desempeño laboral, y cada uno se crea de acuerdo a lo que cada autor considere la fase más adecuada, para poder implementarlo a su personal de área administrativa donde cada autor explica cómo se aplica cada proceso para que tanto el jefe como sus colaboradores revisan y califican los resultados del año, teniendo como base los objetivos establecidos durante la Fase de Planeación. El objetivo de esta fase no es simplemente juzgar el propio desempeño, sino revisar lo que hemos hecho bien, así como aquello que podemos mejorar, para construir una base de datos del éxito y las oportunidades de la Institución.

\section{Conclusiones}

Los modelos de ED ayudan a guiar estratégicamente el Talento Humano (TH) de las Instituciones de Educación Superior IES que es lo más valiosos que poseen, además las 
actividades que le permitan una gestión orientada hacia la obtención de esos buenos resultados que desea, o lo que es lo mismo, la obtención de los objetivos y metas establecidas por la Institución.

\section{Referencias}

ARIAS, F. (2006). El Proyecto de Investigación e Introducción a la Mitología Científica. Caracas: 5ta Edición. Editorial Exísteme.

Casimiro, F. (2007). Los Recursos Humanos en las empresas. Breve análisis en cuanto a evolución y tendencias en su gestión, en Observatorio de la Economía Latinoamericana.

Delhumeau et al. (2013). Modelo de gestión de evaluación del desempeño. Mexico.

García, J. Loredo, E. Luna y M. Rueda. (2008). Memorias del IV Coloquio Iberoamericano sobre la Evaluación de la Docencia. Mexico.

HAYGROUP, F. (2006). Recursos Humanos . Aranzadi.

Ivancevich, J. (2005). Administración de recursos humanos. México: Mc Graw - Hill.

ROBBINS, S. y. (2003). Fundamentos de administración,conceptoy aplicaciones. México.

Seijas , R. (2011). Sistema de Evaluación del desempeño del Recurso Humano. Cumaná.

Villegas, J. (1988). Administración de Personas. Caracas: 1ra. Edición. Editorialtexto s.r.l. .

X. Min-pemg, Zh. Xiao-ho y D. Xina. (2012). Modeling of engineering R\&D staff performances appraisal model base don fuzzy comprehensive evaluation. The 2nd international conference on complexity science \& information engineering: CiVerse ScienceDirect. Procedia system. 